\title{
CONTRÔLE ENDOCRINE DE L'OVOGENÈSE CHEZ LES INSECTES
}

\author{
Marie RAABE \\ Équipe de Neuroendocrinologie des Insectes (C.N.R.S., no 24) \\ associée à l'Université de Paris VI, \\ Laboratoire de Zoologie, Liniversité Paris VI, \\ 11, quai Saint-Bernard, \\ 75005 Paris (France)

\section{RÉSUMÉ}

Le contrôle endocrine de la vitellogenèse des insectes a fait l'objet de nombreuses recherches. Malgré certaines contradictions dans les résultats expérimentaux un schéma général peut être dégagé, qui est présenté ici. Deux facteurs jouent un rôle de première importance : l'un est issu des corpora allata, l'autre des cellules neurosécrétrices du cerveau. Ils agissent en stimulant les synthèses protéiques au niveau du corps gras et en induisant les phénomènes de vitellogenèse au niveau de l'ovaire.

Le déterminisme des premiers stades de l'ovogenèse a été beaucoup moins étudié. Il semble que l'action de l'hormone de mue s'exerçant seule au cours du stade nymphal permette à l'ovaire d'acquérir une certaine compétence et de réaliser des étapes de l'ovogenèse qui précèdent la vitellogenèse.

Si l'on entend le terme d'ovogenèse dans un sens large et si l'on comprend par là les divers phénomènes qui se déroulent depuis la formation de l'ovocyte à partir de l'ovogonie jusqu'au moment de l'ovulation, on peut dire que la question du contrôle endocrine de l'ovogenèse a suscité chez les insectes un grand nombre de travaux. La plupart d'entre eux concernent les phénomènes de vitellogenèse qui seront abordés en premier. Le déterminisme des phénomènes qui précèdent celle-ci ont été beaucoup moins étudiés. Ils seront traités dans la deuxième partie de ce travail. Nous allons donner tout d'abord quelques brèves indications sur l'ovaire des insectes et son fonctionnement. 


\section{L'OVAIRE ET SON FONCTIONNEMENT}

Les ovaires des insectes sont composés d'un nombre variable d'ovarioles formés de deux parties essentielles : le germarium et le vitellarium.

Le germarium se différencie le premier. Il contient les cellules germinales, des cellules mésodermiques qui vont devenir les cellules folliculaires et dans certains cas des cellules sœurs des ovogonies qui joueront un rôle nutritif et que l'on appelle les trophocytes.

Lorsque l'ovogenèse débute il y a multiplication des ovogonies et des cellules folliculaires à la partie basale du germarium. Les ovocytes I se forment et entrent en prophase méiotique, stade auquel ils resteront jusqu'au moment de la fécondation. Ils subissent un certain accroissement puis, s'entourant de cellules mésodermiques et parfois de trophocytes, ils migrent à la partie basale de l'ovariole où ils constituent le premier follicule ovarien du vitellarium. Celui-ci sera formé progressivement de follicules ovariens superposés.

Il existe chez les insectes plusieurs types d'ovaires. Les ovaires panoistiques sont dépourvus de cellules nourricières et chaque follicule ovarien comprend un ovocyte entouré de cellules folliculaires.

Dans les ovaires polytrophiques l'ovocyte reste associé à un certain nombre de cellules sœurs et descend avec elles dans le vitellarium. Les follicules comprennent donc un ovocyte surmonté de trophocytes. Grâce à des liaisons cytoplasmiques, les trophocytes fournissent à l'ovocyte pendant toute la première période de son développement, un apport important de matériaux nutritifs. Ils sont ensuite absorbés par l'ovocyte.

Dans les ovaires acrotrophiques, les follicules se présentent comme dans les ovaires polytrophiques. Les cellules nourricières restent dans le germarium où elles constituent un tissu trophique central. Les ovocytes demeurent unis à celui-ci par de longs ponts cytoplasmiques pendant leur première phase de croissance.

Le vitellarium d'un insecte en période de reproduction occupe une place importante; c'est à son niveau que se réalise la vitellogenèse puis la formation du chorion de l'ovocyte. Lorsque ces phénomènes sont achevés l'ovulation se produit. La fécondation, qui a lieu à l'extrémité du tractus génital, peu avant la ponte, induit la reprise de la méiose.

En dehors du cas de quelques rares espèces vivipares, les œufs des insectes sont volumineux en raison de leur charge en vitellus essentiellement formé par des globules lipidiques et protéiques. La vitellogenèse est un phénomène métabolique très important. Elle fait intervenir différents éléments de l'ovaire. A partir des trophocytes passent dans l'ovocyte de l'ADN, de l'ARN, des protéines, des lipides, des hydrates de carbone et également des mitochondries et des ribosomes. Les cellules folliculaires élaborent de l'ADN, de l'ARN, des protéines, des lipides et du glycogène. L'ovocyte lui-même, notamment dans les ovaires panoïstiques, effectue des synthèses nucléaires (ARN) et cytoplasmiques (lipides, protéines). De plus, il y a passage direct, du sang dans l'ovocyte, par les espaces séparant les cellules folliculaires, d'une ou plusieurs protéines particulières, les protéines femelles. Celles-ci sont synthétisées au niveau 
du tissu adipeux de la femelle en période de reproduction et sont accumulées en grande quantité dans les ovocytes, où elles représentent 75 à $90 \mathrm{p}$. Ioo des protéines vitellines.

\section{CONTRÔLE ENDOCRINE DE LA VITELLOGENÈSE}

Les recherches relatives au contrôle endocrine de la vitellogenèse des insectes ont été effectuées sur des espèces de plusieurs ordres différents. Il convient de remarquer d'emblée que les résultats obtenus, souvent convergents, présentent cependant des contradictions et qu'on tend à penser à l'heure actuelle qu'un schéma unique ne peut s'appliquer à la totalité des insectes. Ceci peut s'expliquer par la grande diversité des modalités de reproduction des insectes et notamment par la façon dont se situent, au cours du développement, les phénomènes primordiaux de stockage des réserves, d'ovogenèse et de vitellogenèse. Enfin, si chez certaines espèces l'activité ovarienne se présente de façon cyclique, chez d'autres, elle est régulière et ne nécessite peut-être pas la présence d'une régulation endocrine.

Il n'est pas possible d'entrer ici dans le détail des travaux, ni de fournir une bibliographie complète $\left.{ }^{(1}\right)$. Les seules données développées seront celles obtenues sur les Criquets Locusta migratoria et Schistocerca gregaria, qui ont fait l'objet des recherches les plus poussées. Celles-ci permettent de formuler un schéma général assez simple qui s'applique dans ses grandes lignes à de nombreuses espèces (fig. I).

Des informations externes et internes variées (photopériode, phérormones excitatrices mâles, copulation, alimentation) sont transmises par les organes sensoriels au cerveau, soit directement, soit par l'intermédiaire de la chaîne nerveuse ventrale. Le cerveau les intègre et élabore dans ses cellules neurosécrétrices deux substances différentes; la sécrétion des cellules A-B et la sécrétion des cellules C (GIRARDIE, I966, Ig67). Lorsque les conditions externes sont favorables, ces deux substances sont déchargées dans l'hémolymphe à partir des corpora cardiaca, organes neurohémaux de stockage des produits de neurosécrétion cérébraux.

La sécrétion des cellules A-B a une action gonadotrope directe sur l'ovaire (Locusta, GIRARDIE, I966 ; BENTZ, I970) et stimule les synthèses protéiques au niveau d'un tissu d'une grande importance métabolique, le tissu adipeux (Schistocerca, HighNam, I962; Highnam et al., I963; HiL.L, I962).

$\mathrm{L}$ a sécrétion des cellules $\mathrm{C}$ joue un rôle gonadotrope indirect : elle active les corpora allata (GIRARDIE, I966, I967). Ces organes sont soumis, par ailleurs, à un contrôle inhibiteur cérébral (STrRoNG, I965; CASSIER, I970).

Les corpora allata, à leur tour, exercent une action gonadotrope (JoLY, I96o ; CASSIER, I964, I965) qui revêt deux aspects différents. Ils interviennent d'une part en contrôlant dans le tissu adipeux la synthèse des protéines nécessaires à la vitellogenèse (MINKS, I967; BENTZ, I970). Ils favorisent, d'autre part, au niveau du follicule, le passage des protéines hémolymphatiques dans l'ovocyte en induisant un élargissement des espaces extracellulaires (BENTZ, I970).

La Blatte vivipare, Leucophaea maderae, qui a suscité également de nombreuses recherches, permet d'aboutir à un schéma voisin. Le contrôle exercé par le cerveau

(1) Les lecteurs pourront se référer à la mise au point de Cassier (1967) et aux ouvrages de JoLy (I968) et ENGELMANN (I970). 


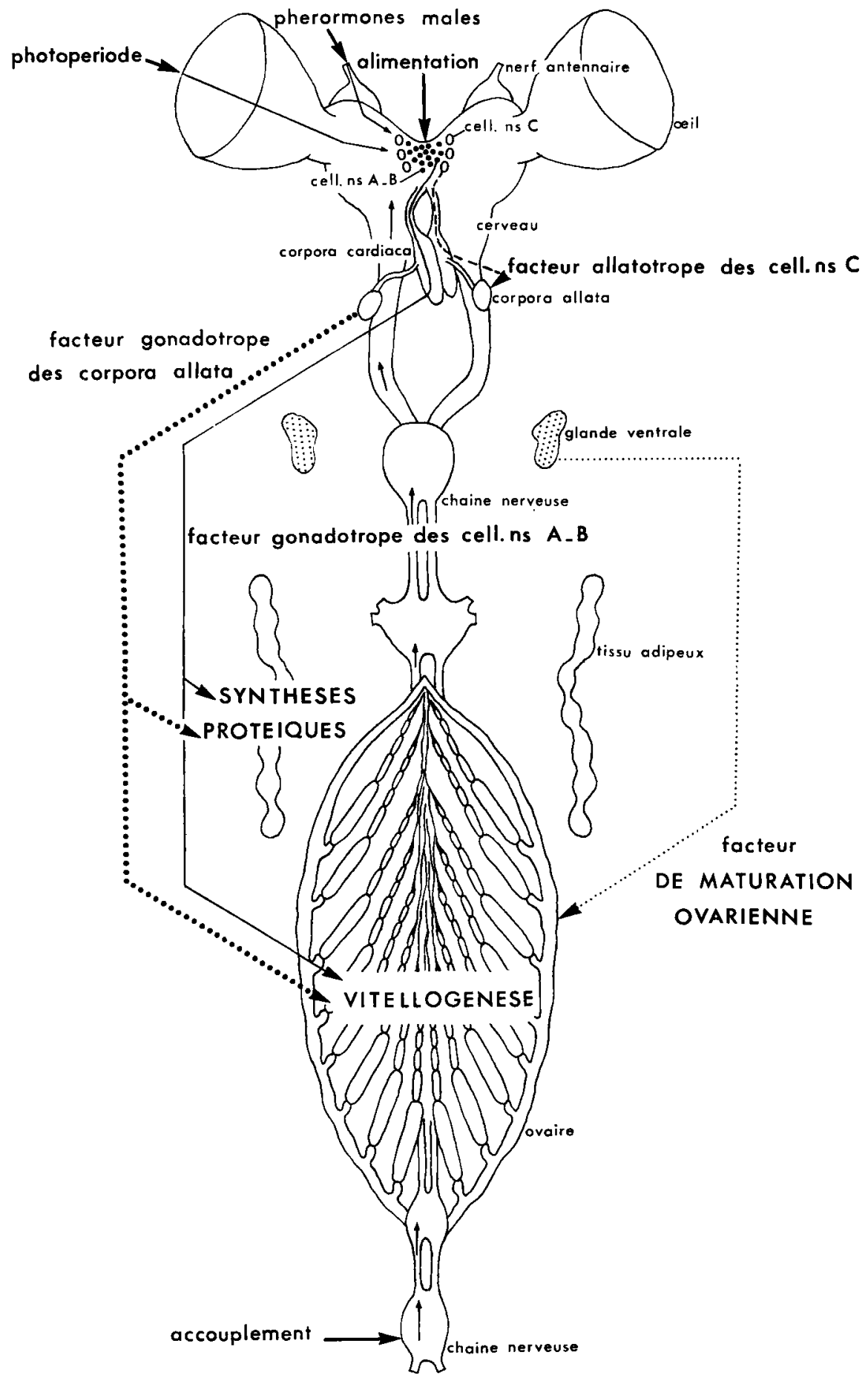

FIG. I. - Représentation schématique du contróle de l'ovogenèse chez les Acridiens

(Dessin en partie d'après RAABE (I964) et CASSIER (r967), modifié) de mue.

cell. ns : cellules neurosécrétrices. La glande ventrale est considérée comme homologue des glandes 
sur les corpora allata, de nature inhibitrice, s'effectue par voie nerveuse et non neurosécrétoire. Une section des nerfs cérébro-allates provoque donc une activation des corpora allata se manifestant par une augmentation de volume de ces organes et une reprise de la vitellogenèse pendant la période de repos ovarien qui accompagne la gestation (ENGELMANN, I957 a).

Des discussions nombreuses ont eu lieu à propos du rôle gonadotrope ou métabolique joué par le cerveau et les corpora allata, les synthèses protéiques étant sous le contrôle de l'un ou de l'autre de ces organes selon les auteurs. En rapprochant les travaux récents obtenus sur plusieurs espèces il semble que le cerveau et les corpora allata possèdent les deux actions ce qui n'est pas surprenant car ils contrôlent divers aspects de la reproduction et non seulement la vitellogenèse.

Les mécanismes endocrines mis en évidence chez les Criquets ne semblent pas exister chez toutes les espèces. Ainsi, parmi les Lépidoptères, certains présentent un contrôle classique (KARLINSKY, I962, I963), alors que chez d'autres la vitellogenèse semble se faire indépendamment des corpora allata (BounhIoL, I936; WILIIAMs, I952). Il faut rappeler ici que le facteur gonadotrope des corpora allata est identique au facteur juvénile de ces organes qui a une grande importance dans la croissance des insectes. Au cours de celle-ci sa présence conditionne le maintien des caractères larvaires, alors que son absence, au stade nymphal, permet la métamorphose. On peut constater que les espèces où la vitellogenèse est indépendante des corpora allata sont précisément celles où la maturation des ovocytes se déroule au stade nymphal, c'està-dire dans un milieu intérieur ne pouvant contenir l'hormone juvénile ou gonadotrope. A une physiologie différente correspond apparemment un déterminisme endocrine différent.

\section{CONTRÔLE ENDOCRINE DES PREMIERS STADES DE L'OVOGENÈSE}

On s'est souvent demandé pourquoi l'ovaire était incapable de fonctionner chez les larves puisque celles-ci étaient riches en hormone des corpora allata et la notion de puberté a été formulée (JoL, I945; JoL, I960). Selon cette conception l'ovaire devrait pour pouvoir se développer passer par une étape particulière réalisée au cours de la vie nymphale.

Les expériences d'ITTYCHERIAH et NAYAR (I968), sur une punaise, Iphita, ont démontré le bien-fondé de ce point de vue. Elles ont consisté en transplantations d'ovaires de stades divers. Si l'on implante à des femelles adultes des ovaires provenant de larves de stade 3,4 ou de nymphes du début du stade 5 , on n'observe aucun développement (fig. 2 , exp. I, 2, 3). Par contre, si les ovaires sont prélevés sur des nymphes en fin de stade 5 (fig. 2, exp. 4) on assiste au développement ovarien. Le milieu intérieur de la nymphe âgée a donc une action décisive. Il est caractérisé, comme les autres stades larvaires par la présence de l'hormone de mue mais diffère de ceux-ci par l'absence de l'hormone juvénile. Deux autres expériences confirment les premières (fig. 2, exp. 5 et 6 ). Si des ovaires de larves de stade 3 sont implantés pendant un certain temps dans des nymphes du début du stade 5, puis dans des larves de stade 4 , on n'observe aucune modification ovarienne. Par contre, s'ils sont implantés dans des nymphes de la fin du stade 5 , puis dans des larves de stade 4 , il se produit 


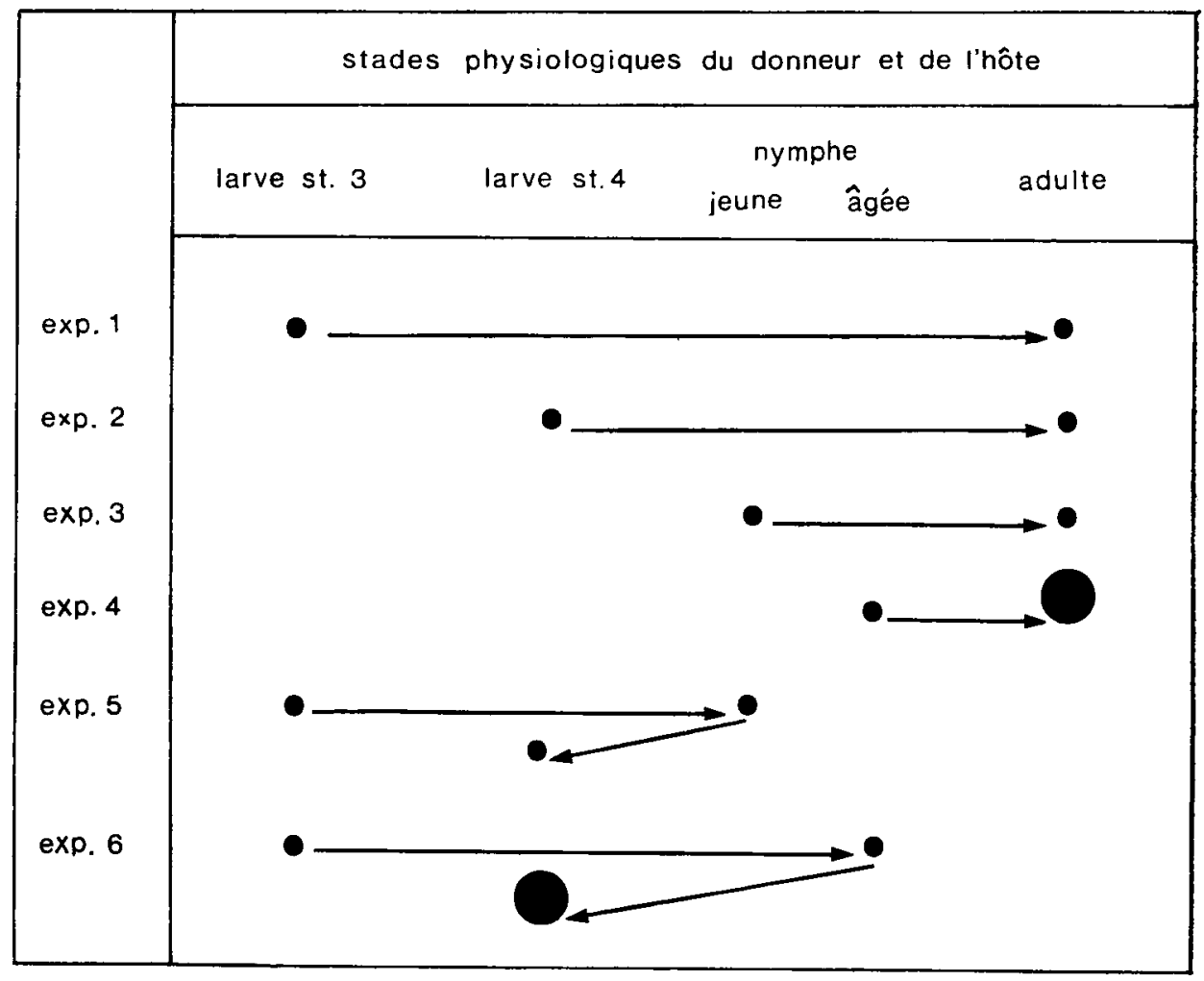

FIG. 2. - Représentation schématique des transplantations ovariennes effectues par ItTycheriah et Nayar sur la Punaise, Iphita limbata

Le point d'origine des flèches indique le stade du donneur; leur point d'arrivée représente le stade de l'hôte définitif ou temporaire. Les petits points noirs correspondent à des ovaires demeurant infantiles; les gros points noirs à des ovaires en voie de développement.

une maturation ovarienne comprenant la différenciation de la zone trophique du germarium et l'apparition des ovocytes qui migrent dans le vitellarium. L'ovaire peut donc se développer en milieu larvaire à condition d'avoir acquis une certaine compétence qui résulte de son séjour dans un milieu riche en hormone de mue mais dépourvu d'hormone juvénile.

Des expériences réalisées sur Locusta donnent des résultats plus complexes mais indiquent également que le séjour dans un milieu contenant uniquement 1'hormone de mue est nécessaire à la maturation ovarienne (STRICH-HALBWACHS, I958; JOLY, Ig60 ; VOGEL, I969).

L'intervention de l'hormone de mue dans les premiers stades de l'ovogenèse a été démontrée également en culture d'organes sur l'ovaire nymphal du Coléoptère Tenebrio molitor (LAVERDURE, I969, I970, I97I). Alors qu'aucune évolution n'est obtenue sur un milieu de base, on observe, après adjonction d'ecdysone, hormone stéroïde longtemps considérée comme originaire des glandes de mue (1), une croissance

(1) On pense actuellement que l'ecdysone pourrait être élaborée ailleurs que dans les formations désignées sous le nom général de glandes de mue. Il faut noter cependant que dans de nombreuses expériences on obtient des effets similaires par injection d'ecdysone ou implantation de glande de mue. 
des ovocytes et une différenciation des ovarioles. Cette action est toutefois paralysée par un apport d'une substance mimétique de 1'hormone des corpora allata, ce qui est conforme aux notions mises en évidence plus haut.

Les résultats de LAVERDURE ont été confirmés récemment sur un Lépidoptère, Galleria mellonella (ShrbuYA et YAGr, I972). La mise en culture en présence d'ecdystérone d'ovaires du dernier stade larvaire de cette espèce est suivie d'une différenciation des constituants du germarium (ovocytes, trophocytes, cellules folliculaires) et de l'organisation du vitellarium.

L'hormone de mue (hormone des glandes de mue ou ecdysone) semble donc bien stimuler les premiers processus d'ovogenèse. Dans la suite du développement ovarien au cours de la vitellogenèse, son action s'inverse et devient inhibitrice, comme le montrent les expériences d'implantation de glande de mue aux adultes réalisées sur la Blatte (Engelmann, r957), le Grillon (Thomas, I964), le Criquet (Cassier, I970), et les expériences d'ingestion d'ecdysone et de ses analogues effectuées sur la Mouche domestique et le Coléoptère, Tribolium confusum (RoBbins et al., I968).

Un travail récent sur Locusta migratoria (JoL, I, I7I) met bien en évidence la double action de la glande de mue (glande ventrale), qui chez l'adulte, stimule la croissance des petits ovocytes et provoque la dégénérescence des grands.

\section{CONCLUSION}

Chez les Insectes les premiers stades de l'ovogenèse semblent dépendre de l'action isolée de l'hormone de mue. Les phénomènes de vitellogenèse sont contrôlés à la fois par les cellules neurosécrétrices cérébrales et par les corpora allata.

\section{SUMMARY}

ENDOCRINE CON'TROL, OF OOGENESIS IN INSECTS

The endocrine control of vitellogenesis has been intensively studied in insects. Despite some discrepancies in the experimental results, a general picture may be drawn. Two factors play a major role, one originating from the corpora allata, the other from the neurosecretory cells of the brain. They stimulate protein synthesis in the fat body and vitellogenesis in the ovary.

The control of the first stages of oogenesis has been less studied. It seems that the molting hormone acting alone during the nymphal stage enables the ovary to develop further, and induces the oogenesis stages which preceed vitellogenesis.

\section{RÉFÉRENCES BIBLIOGRAPHIQUES}

Bentz F., 197o. Contribution à l'étude de la vitellogenèse chez Locusta migratoria L. (Orthoptère. These de $3^{\text {e }}$ cycle, Strasbourg.

Bentz F., Girardie A., 1969. Action de la pars intercerebralis et des corpora allata sur les proteines ovariennes de Locusta migratoria migratorioïdes (Orthoptere) au cours de la vitellogenèse. C. R, Acad. Sci., F4., 269, 2014-2017. 
Bovnhtol J.-J., I936. Métamorphose après ablation des corpora allata chez le ver à soie Bombyx mori L. C. R. Acad. Sci., Fr., 203, 388-389.

Cassier P., 1964. Effets immédiats et transmis des implantations de corps allates sur la fécondité et la descendance des femelles de Locusta migratoria migratorioïdes (R. et F.); phase grégaire (Insecte Orthoptéroïde). C. R. Acad. Sci., Fr., 259, 2706-2708.

CAssier P., I965. Déterminisme endocrine de quelques caractéristiques phasaires chez Locusta migratoria migratorioïdes (R. et F.). Insectes soc., Fr., 12, $71-80$.

CAssier P., I967. La reproduction des Insectes et la régulation de l'activité des corps allates. Ann. biol., Fr., 4 (II-I2), 595-67o.

Cassier P., I97o. Influence des conditions d'élevage (isolement et groupement) sur la fécondité des femelles de Locusta migratoria migratorioïdes (R. et F.) et sur les caractéristiques de leur descendance : données endocrines. Coll. Int. C. N.R.S., 189, 87-1 II.

Engelmann F., I957a. Die Steuerung der Ovarfunktion bei der ovoviviparen Schabe Leucophaea maderae (FABR.). J. Insect Physiol., G.-B., 1, 257-278.

Engelmann F., I957 b. Hemmung der Eireifung durch Prothoraxdrüsen bei Leucophaea maderae (Orthoptera). Natureissenschaften, Dtsch., 76 (6), 722-740. 722-740.

ENGELMANN F., I970. The physiology of insect reproduction, 44, 143-I89. In International series of monographs in pure and applied biology; division zoology. General editor: G. A. Kerkut, Pergamon Press.

GrRardie A., I966. Contrôle de l'activité génitale chez Locusta migratoria. Mise en évidence d'un facteur gonadotrope et d'un facteur allatotrope dans la pars intercerebralis. Bull. Soc. zool., Fr., 91, 423-439.

GrRARdie F., 1967. La pars intercerebralis chez Locusta migratoria L. (Orthoptère) et son rôle dans le developpement. These. Strasbourg.

Highnam K. C., 1962. Neurosecretory control of ovarian development in Schistocerca gregaria gregaria. Quart. J. micr. Sci., G.-B., 103, 57-72.

Highnam K. C., Lusis O., HiLl L., I963. Factors affecting oocyte resorption in the desert locust Schistocerca gregaria (FoRskÅL). J. Insect Physiol., G.-B., 9, 827-837.

Hill L., I962. Neurosecretory control of haemolymph protein concentration during ovarian development in the desert locust. J. Insect Physiol., G.-B., 8, 609-6rg.

ITTYChERIAH P. I., NAYAR K. K., r967. Ovarian response to corpus allatum in Iphita limbata STAL. Curr. Sci, India, 22, 608-609.

Joly L., I96o. Fonction des corpora allata chez Locusta migratoria (L.). Thèse, Strasbourg.

Joly L., r97I. Action de l'implantation des glandes ventrales sur l'activité génitale des imagos de Locusta migratoria L. C. R. Acad. Sci., Fr., 272, 2326-2329.

Jory P. I945. La fonction ovarienne et son contrôle humoral chez les Dytiscides. Arch. Zool. expér. gen., Fr., 84, 47-I64.

Joly P., r968. Endocrinologie des Insectes, 7, 209-252 ; G. P. B., Masson et Cie.

KARLinsky A., r962. Effets de l'ablation des corpora allata larvaires sur le développement ovarien de Pieris brassicae L. (Lépidoptère). C. R. Acad. Sci., Fr., 255, I9I-I93.

KARLINSKY A., I963. Effets de l'ablation des corpora allata imaginaux sur le développement ovarien de Pieris brassicae L. (Lépidoptère). C. R. Acad. Sci., Fr., 256, 4IoI-4103.

Laverdure A.-M., I969. Culture in vitro de l'ovaire nymphal de Tenebrio molitor (Coléoptère) en présence d'ecdysone. C. R. Acad. Sci., Fr., 269, 8z-85.

LAVERdure A.-M., r970. L'évolution de l'ovaire chez la nymphe et l'adulte de Tenebrio molitor (Coléoptère). La vitellogenèse. Thèse, Orsay.

LAverdure A. M., I97I. Étude des conditions hormonales nécessaires à l'évolution de l'ovaire chez la nymphe de Tenebrio molitor (Coléoptère). Gen. Comp. Endocrinol., U. S. A., 17, 467-478.

Minks A. K., I965. Haemolymph protein and amino-acid composition as influenced by the corpus allatum in Locusta migratoria migratoriożdes (R. et F.). Kkl. nederl. Akad. Wetensch., Proc., C, 4, 320-323.

RAABE M., r964. Nouvelles recherches sur la neurosécrétion chez les Insectes. Ann. Endocrinol., Fr., 25, I07-I12.

Robrins W. E., Kaplanis J. S., Thompson M. J., Shortino T. J., Cohen C. F., Joyner S. C., I968. Ecdysones and analogs : effects on development and reproduction of Insects. Science, U.S.A., 161, I I 58-I I60.

Shibuy I., YAGI S., 1972. Effects of ecdysterone on cultivated ovaries of the greater wax moth larvae (Lepidoptera: Pyralidae). Appl. Ent. Zool., U.S. A., 7, 97-98.

Strich-Halbwachs M. C., I958. Action de la glande ventrale sur le développement ovarien de Locusta migratoria L. (Orthoptera). J. Insect Physiol., G.-B., 1, 346-35I.

Strong L., 1965. The relationships between the brain, corpora allata and oocyte growth in the Central American Locust, Schistocerca Sp. II. The innervation of the corpora allata, the lateral neurosecretory complex and oocyte growth. J. Insect Physial., G.-B., 11, 27I-280. 
Tromas A., 1964. Etude expérimentale relative au contrôle endocrine de l'ovogenèse chez Gryllus domesticus L. Bull. Soc. zool., Fr., 89 (5-6), 835-854.

VoGri A., rg69. Mise en évidence de la phase de déclenchement du développement ovarien chez Locusta migratoria L. C. R. Acad. Sci., Fr., 268, Ir94-II96.

Williams C. M., 1952. Physiology of insect diapause. IV. The brain and protoracic glands as an endocrine system in the Cecropia silkworm. Biol. Bull., U.S. A., 108, 120-138. 